\title{
Emulation of utility functions over a set of permutations: sequencing reliability growth tasks
}

\author{
Kevin J Wilson, Daniel A Henderson \\ School of Mathematics and Statistics, Newcastle University, UK \\ and \\ John Quigley \\ Department of Management Science, University of Strathclyde, UK
}

August 29, 2017

\begin{abstract}
We consider Bayesian design of experiments problems in which we maximise the prior expectation of a utility function over a set of permutations, for example when sequencing a number of tasks to perform. When the number of tasks is large and the expected utility is expensive to compute, it may be unreasonable or infeasible to evaluate the expected utility of all permutations. We propose an approach to emulate the expected utility using a surrogate function based on a parametric probabilistic model for permutations. The surrogate function is fitted by maximising the correlation with the expected utility over a set of training points. We propose a suitable transformation of the expected utility to improve the fit. We provide results linking the correlation between the two functions and the number of expected utility evaluations to undertake. The approach is applied to the sequencing of reliability growth tasks in the development of hardware systems, in which there is a large number of potential tasks to perform and engineers are interested in meeting a reliability target subject to minimising costs and time. An illustrative example shows how the approach can be used and a simulation study demonstrates the performance of the approach more generally.
\end{abstract}

Keywords: Multi-attribute utility, Bayesian design of experiments, design for reliability, Benter model 


\section{Introduction}

The maximisation of the prior expectation of a utility function to find the optimal design of an experiment in a Bayesian analysis is challenging as a result of the necessity of evaluating the expected utility for all designs. We can make progress by using an approximation based on a surrogate function which is faster to evaluate, known as an emulator [Kennedy and O'Hagan, 2001, Henderson et al., 2009, Young and Ratto, 2011, Zhou et al., 2011]. When the possible experimental designs are a set of permutations this adds complexity as commonly used emulators, such as Gaussian process emulators, are based on the assumption of a smooth relationship between the inputs to, and outputs from, the function being emulated, which is unlikely to be true in this case.

An example is the reliability growth of hardware products under development. During development, initial designs are subject to detailed analysis, identifying improvements until performance requirements are met. Reliability tasks which analyse the design and facilitate the enhancement include fault tree analysis, failure modes and effects analysis and highly accelerated life testing. These tasks can be resource intensive and costly to implement and each has the goal of design improvement by understanding weaknesses. Multiple tasks may uncover the same fault in the current design of the product and some faults may not be uncovered by any of the tasks. Therefore, the engineers are faced with the decision problem of which tasks to perform and in what order to grow their reliability to the required level. Their possible experimental designs are the permutations of the possible reliability tasks to carry out.

\subsection{Emulation of permutations}

The use of surrogate models for emulating expensive functions dates back at least as far as Sacks et al. [1989] who propose a Gaussian process prior for the output of a complex computer model. An early example of emulating an expensive utility function in the context of Bayesian optimal design is described in Müller and Parmigiani [1995]. The use of surrogate models for emulating an expensive function over a set of permutations has received relatively little attention. An early example is described in Voutchkov et al. [2005] in which a surrogate model is proposed for ordering a sequence of welding tasks in the aircraft industry. Most surrogate models for functions with continuous input spaces, such as Gaussian processes or radial basis functions, are based on the premise that points close in input space will lead to similar values of the expensive function. In terms of a generic approach to the problem, a natural first step for emulating a function over permutations would be 
to replace the Euclidean distance between inputs in surrogate models with a more natural distance measure on the space of permutations. This is the approach proposed by Moraglio and Kattan [2011], Moraglio et al. [2011] and Kim et al. [2014]. These authors focused on a radial basis function-based surrogate model for general combinatorial input spaces, including permutations. They used the Hamming distance and Kendall tau distance as two distance measures on sets of permutations; see Marden [1995] for details.

This approach of replacing the Euclidean distance with a permutation-based distance was generalised by Zaefferer et al. [2014b] to a Gaussian process-based surrogate model. The use of a Gaussian process-based surrogate allowed the authors to use the efficient global optimisation approach of Jones et al. [1998] and a standard Kriging estimator. The authors reported improved performance over a radial basis function-based surrogate on a set of combinatorial optimisation problems. This work was extended by Zaefferer et al. [2014a] who investigated 14 different distance functions over sets of permutations for Gaussian process-based surrogates. An approach based on choosing the distance function by maximum likelihood yielded the best results, with the Hamming distance the best single distance measure. Zaefferer [2015] describes an R package 'CEGO' for implementing the Gaussian process-based surrogate modelling and optimisation approach that is proposed in Zaefferer et al. [2014b] and Zaefferer et al. [2014a].

\subsection{Design for reliability}

Design for reliability principles are not sufficient for designing complex systems [Wayne and Modarres, 2015, U.S. Department of Defense, 2011, 2008] and as such reliability growth continues to provide a key role in product development. However, achieving growth through Test Analyse And Fix (TAAF) is expensive which leads organisations to develop integrated programs of activities to reduce reliance on testing [Krasich et al., 2004]. There are several activities employed to enhance reliability during design and development, e.g. Highly Accelerated Life Testing (HALT), Fault Tree Analysis, Failure Modes and Effects Analysis (FMEA) (see Blischke and Murthy [2011]), each of which requires resources and must be managed, see for example IEC 61014. While these activities have different perspectives upon a design, each has the common goal of seeking to improve the design. To date, little attention has been paid in the literature on optimally sequencing activities to achieve target reliability while minimising costs.

Past research has focused primarily on either determining optimal designs, for example with levels of redundancy [Levitin et al., 2015, Caserta and Voß, 2015], or modelling the fault detection 
rate from TAAF tests, for which there is international standard IEC 61164, comprising both frequentist and Bayesian methods [Crow, 1974, Walls and Quigley, 1999, Quigley and Walls, 2003]. Research at the program level is scarce, either focusing on monitoring progress graphically [Krasich, 2015, Walls et al., 2005] or project management issues such a metrics, infrastructure and documentation [Eigbe et al., 2015]. While minimising program costs has been explored by Hsieh and Hsieh [2003], Hsieh [2003] a key shortcoming has been the representation of growth and program costs through continuous functions as decisions are discrete and choices are finite [Guikema and Pate-Cornell, 2002]. An exception to this is Johnston et al. [2006] who explored minimising program cost with discrete choices through integer programming methods and Wilson and Quigley [2016] who provided a solution incorporating multi-attribute utility functions to allow trade-offs between attributes; however neither paper addressed the issue of sequencing the activities.

Table 1 provides a simplified illustrative example which is similar to practice but substantially reduced in size and presented without industrially sensitive design details. Table 1 presents five engineering concerns with the design of an aerospace system and four development activities; these were identified through an elicitation process.

\begin{tabular}{|c|cccc|}
\hline Concern & Salt and Fog & Vibration Test & Electrical & Highly Accelerated \\
& Stress Test & Stress Test & Life Test (HALT) \\
\hline Temperature control & & & \\
Moisture Ingress & & & \\
Difference in TCE & & & \\
Circuitry voltage spike & & & \\
Impedance & & & & \\
\hline
\end{tabular}

Table 1: Illustrative efficacy matrix with 5 concerns and 5 activities

The ability of the design to maintain its temperature within acceptable limits was a concern for the engineers, and while the current design may achieve desired control, the engineers were uncertain. Moisture ingress was a concern as new material was being used in the design compared with heritage designs and, while preliminary tests indicated that the new material would not be deficient in this regard, the engineers were uncertain. The significance of the difference of the Temperature Coefficient of Expansion (TCE) between different materials was a concern for aspects of the system as this may prove to be a deficiency in the design. The possibility of aspects of the circuitry generating unacceptable voltage spikes was identified. The final concern listed related to uncertainty between the soldering, the environment and the circuits, where residue remaining on solder joint could cause failure for high impedance circuits in environments of high moisture. 
Associated with each concern is a probability that the concern is a fault, i.e. if the system were used in operation it would fail due to an imperfection or deficiency described by the concern.

There are a variety of reliability tests that can be conducted to assess an item's design at the component, sub-assembly or system level; these include life and environment tests, for example Highly Accelerated Life Testing (HALT), as well as environmental testing against specific stresses such as vibration, heat, electricity, salt, fog or moisture. For a more detailed discussion see O'Connor and Kleyner [2012] or Silverman [1998]. Table 1 presents four such activities and within each cell of the table we would input a probability measuring how likely the associated activity would be to identify the associated concern as a fault, assuming that the concern was a fault, i.e. the probability it does not slip through test undetected.

Once populated with probabilities, efficacy matrices can help identify if there are concerns with no associated activities and can form the basis of a reliability growth programme. Typically, the matrices have a many to many relationship, where a fault can be exposed by several activities and an activity can expose several faults. As such, there are choices to be made with respect to which activities should be scheduled, with the aim of either identifying the faults in the design or providing evidence that the concern is not a fault. While we have presented a simple example, a large system design may have hundreds of concerns with as many activities.

\subsection{This paper}

In this paper we initially consider the problem of emulating the prior expectation of a utility function over a set of permutations. We propose an approach to perform the emulation using the Benter model [Benter, 1994] as the basis for the surrogate function. The Benter model is an extension of the Plackett-Luce model [Plackett, 1975, Luce, 1959], a popular probabilistic model for permutations; see [Marden, 1995] for further background on probabilistic models for permutations. We outline how to fit the Benter model based on a training sample of model runs for the expected utility and propose suitable transformations of both the expected utility and surrogate function to improve the fit. We give results which provide us with a method of deciding on the number of evaluations of the expected utility to make based on the optimal values of the surrogate function.

We consider the problem of sequencing reliability growth tasks specifically. We adapt a commonly used model for reliability growth [Johnston et al., 2006, Quigley and Walls, 2006] under development to incorporate explicitly uncertainty on the reliability function and utilise this to assess the cost and time of performing any sequence of reliability tasks, taking into account that we 
will stop testing when we reach a reliability target. We propose a two-attribute utility function to solve the design of experiments problem.

In Section 2 we outline our general approach to emulation of the expected utilities on sets of permutations and give theoretical results. In Section 3 we consider the model for reliability growth and develop the solution to the decision problem. In Section 4 we give an illustrative example informed by work with industrial partners in the aerospace industry. Section 5 considers two simulation studies to examine the strengths and weaknesses of the emulator more generally and provide guidance on the choice of training and evaluation set sizes for the emulator. In Section 6 we summarise and give some areas for further work.

\section{Emulation of utility functions on permutations}

\subsection{The emulator}

Suppose that there are $J$ tasks which are to be performed in a sequence. Then there are $J$ ! possible sequences, or permutations, of the $J$ tasks. Let $\mathcal{S}^{J}$ denote the set of all permutations of the $J$ tasks. Each sequence of tasks $\boldsymbol{x}=\left(x_{1}, x_{2}, \ldots, x_{J}\right) \in \mathcal{S}^{J}$, where $x_{k}$ denotes the $k$ 'th task, gives rise to an expected utility $u=U(\boldsymbol{x}) \in[0,1]$, where 0 represents the least preferable possible outcome and 1 represents the most preferable possible outcome.

If we can compute $U(\boldsymbol{x})$ for all possible sequences then we can solve the system design problem by choosing the sequence which maximises the expected utility. However, it is often the case that $U(\boldsymbol{x})$ is time consuming to compute so that evaluating the expected utility for each possible permutation $\boldsymbol{x}$ may not be feasible. Instead, we can treat $U(\cdot)$ as an expensive deterministic function and try to "emulate" it using a less expensive surrogate, with the idea being that it is feasible to evaluate the surrogate function at all possible permutations in reasonable time, or allow us to explore the space of permutations more efficiently.

Thus we wish to find a surrogate function $f(\cdot)$ which takes a sequence $\boldsymbol{x}$ as input and outputs a real scalar quantity such that $f\left(\boldsymbol{x}_{i}\right)>f\left(\boldsymbol{x}_{j}\right)$ if $U\left(\boldsymbol{x}_{i}\right)>U\left(\boldsymbol{x}_{j}\right)$, for $i \neq j$.

Our proposed surrogate function,

$$
f(\boldsymbol{x}) \equiv f(\boldsymbol{x} ; \boldsymbol{\theta}, \boldsymbol{\alpha})=\sum_{j=1}^{J} \alpha_{j} \log \left(\theta_{x_{j}}\right)-\log \left(\sum_{m=j}^{J} \theta_{x_{m}}^{\alpha_{j}}\right)
$$

is derived from the loglikelihood function of the Benter model [Benter, 1994] which we treat as a 
function of $\boldsymbol{x}$ for fixed values of the positive parameters $\boldsymbol{\theta}=\left(\theta_{1}, \ldots, \theta_{J}\right)$ and $\boldsymbol{\alpha}=\left(\alpha_{1}, \ldots, \alpha_{J}\right)$. Note that when $j=J$ the contribution to $f(\boldsymbol{x})$ is 0 , and so we cannot estimate $\alpha_{J}$; we therefore set $\alpha_{J}=0$. The Benter model is an example of a multistage ranking model [Fligner and Verducci, 1988] and was proposed by Benter to overcome perceived deficiencies of the Plackett-Luce (PL) model [Plackett, 1975, Luce, 1959] for analysing the results of horse races. Benter introduced a parameter $\alpha_{j}$ for each "stage", to reflect its importance. The stage-dependent flexibility of the Benter model has been used in several applications, such as the analysis of voting data [Gormley and Murphy, 2008]. A more detailed description of the Benter model, together with other extensions of the PL model, such as its reversed version, the reverse Plackett-Luce (RPL) model, is given in Mollica and Tardella [2014]. The PL model is obtained from the Benter model by setting $\alpha_{j}=1$ for $j=1,2, \ldots, J$. We are using the parametric structure of the Benter model simply as a surrogate $f(\boldsymbol{x})$ for $U(\boldsymbol{x})$ and we are not implying or assuming that the expected utility is a probability. As well as displaying good empirical performance, which we detail in Section 5, the parametric structure of the Benter model does have some properties that may be desirable for emulating the expected utility function of a set of sequences of tasks. For example, for fixed values of $\boldsymbol{\alpha}$, the positive parameter $\theta_{k}$ is associated with task $k \in\{1,2, \ldots, J\}$, such that $\theta_{k}$ is proportional to the utility when task $k$ is scheduled first. Similarly, for fixed $\boldsymbol{\theta}$, the positive parameter $\alpha_{\ell}$ is associated with position $\ell$ in the sequence of tasks, and can be thought of as reflecting the relative importance of the task that is performed $\ell$ th in the sequence. We might expect the $\alpha$ parameters to be larger for the tasks that are performed early on in the sequence than those performed later in the sequence, when the target reliability may already have been reached.

We would like to choose parameters $\boldsymbol{\psi}=\{\boldsymbol{\theta}, \boldsymbol{\alpha}\}$ such that sequences $\boldsymbol{x}$ with high expected utility $U(\boldsymbol{x})$ also have high values of $f(\boldsymbol{x})$. To do so, we choose $\boldsymbol{\psi}$ to maximise the correlation between the logit transformed expected utilities from a training sample, $\boldsymbol{x}_{1}, \ldots, \boldsymbol{x}_{N}$, for some $N<<J$ !, and their values under the surrogate $f(\boldsymbol{x})$. Note that any appropriate correlation function may be used, e.g. Pearson, Spearman, Kendall. Similarly we place no restrictions on the choice of training sample, but defer discussion of choices of training sample to Section 6. Specifically, let the vector of expected utilities of the training sample be $\boldsymbol{u}=\left(u_{1}, \ldots, u_{N}\right)$, where $u_{i}=U\left(\boldsymbol{x}_{i}\right)$, for $i=1,2, \ldots, N$; as we observe expected utilities close to zero and one, we work with the logit transformed values of the expected utility, $\eta_{i}=\log \left(u_{i} /\left(1-u_{i}\right)\right)$. Also, let the vector of surrogate function values of the training sample be $\boldsymbol{f}^{\boldsymbol{\psi}}=\left(f_{1}^{\boldsymbol{\psi}}, \ldots, f_{N}^{\boldsymbol{\psi}}\right)$, where $f_{i}^{\boldsymbol{\psi}}=f\left(\boldsymbol{x}_{i} ; \boldsymbol{\psi}\right)$ for $i=1, \ldots, N$ 
with $f(\cdot ; \cdot)$ as defined in Equation (1). Thus, we seek $\boldsymbol{\psi}$ to maximise $c(\boldsymbol{\psi})=\operatorname{Cor}\left(\boldsymbol{\eta}, \boldsymbol{f}^{\psi}\right)$, i.e.,

$$
\hat{\psi}=\operatorname{argmax}_{\psi} c(\psi)
$$

where $\boldsymbol{\eta}=\left(\eta_{1}, \ldots, \eta_{N}\right)$. The maximisation of the correlation can be performed using the NelderMead simplex algorithm [Nelder and Mead, 1965] as implemented in the optim function in $R$ [R Core Team, 2014]. We run the optimisation from a small number of different starting points (usually 5), and choose the value of $\psi$ which gives the maximum correlation.

Our initial emulator of the logit transformed expected utility function is therefore our surrogate function evaluated at $\hat{\boldsymbol{\psi}}$, that is $\hat{f}(\cdot)=f(\cdot ; \hat{\boldsymbol{\psi}})$.

\subsubsection{Regression-adjusted surrogate model}

The emulator $\hat{f}(\cdot)=f(\cdot ; \hat{\psi})$ performs well empirically, as detailed in Section 5 , but it can be improved by an adjustment based on simple linear regression. We fit a linear model with response vector $\boldsymbol{\eta}$ and linear predictor $\beta_{0}+\beta_{1} \hat{f}(\boldsymbol{x})+\beta_{2} \hat{f}(\boldsymbol{x})^{2}+\beta_{3} \hat{f}(\boldsymbol{x})^{3}$ using ordinary least squares, where $\hat{f}(\boldsymbol{x})$ is the fitted value of the surrogate function under the Benter model. The resulting fitted mean function

$$
f^{\star}(\boldsymbol{x})=\hat{\beta}_{0}+\hat{\beta}_{1} \hat{f}(\boldsymbol{x})+\hat{\beta}_{2} \hat{f}(\boldsymbol{x})^{2}+\hat{\beta}_{3} \hat{f}(\boldsymbol{x})^{3}
$$

is our regression-adjusted emulator. This regression-adjusted surrogate improves upon $\hat{f}(\cdot)$ in several ways. Firstly, it is easier to interpret the output from $f^{\star}(\cdot)$ as it is on the same scale as the logit-transformed expected utilities (although we emphasise that this is not an essential feature of an emulator as all we are interested in is the relative order of the expected utilities). Secondly, the relationship between logit expected utility and the surrogate function is made more linear, which aids interpretation. Thirdly we may obtain a quantification of the uncertainty in the emulator output for a given sequence $\boldsymbol{x}$ through the usual linear model-based prediction intervals, if desired. Such prediction intervals are necessarily conditional on the estimated surrogate model parameters $\hat{\psi}$; taking into account the uncertainty in $\hat{\psi}$ would lead to wider intervals. Note that if an emulator for the expected utility $U(\cdot)$ (rather than the log-transformed expected utility) is required then we simply take

$$
f^{\dagger}(\boldsymbol{x})=\frac{\exp \left\{f^{\star}(\boldsymbol{x})\right\}}{1+\exp \left\{f^{\star}(\boldsymbol{x})\right\}}
$$

though such a transformation is not essential. 


\subsection{Properties of the emulator}

Unless there is a perfect correspondence between $U(\boldsymbol{x})$ and $f(\boldsymbol{x})$, there is no guarantee that the optimal sequence under the surrogate function, $\hat{\boldsymbol{x}}$, will be the sequence which maximises the expected utility. In this case, we can use $f(\cdot)$ to propose a set of $M$ candidate sequences which may have a high expected utility, specifically those which maximise $f(\cdot)$. We can then take the sequence out of the $N+M$ evaluations with the largest expected utility as our best estimate of the optimal sequence.

The following results allow us to link the correspondence between $U(\boldsymbol{x})$ and $f(\boldsymbol{x})$ and the probability of observing the sequence with maximum expected utility in the $M$ candidate sequences. The proof is given in the Supplementary Material.

Proposition 1. If we have $J$ tasks with $R=J$ ! permutations, then the sequence of tasks with highest expected utility will be in the $M$ sequences with highest value of the function $f(\cdot)$ with probability

$$
\sum_{m=1}^{M} \frac{N_{R-1, \delta-m+1}}{N_{R, \delta}},
$$

where $\delta$ is the Kendall's tau distance between the utility and $f(\cdot)$ for all possible sequences, $N_{R, \delta}$ is given by $N_{R, \delta}=C_{R, \delta}-C_{R, \delta-1}$, and $C_{R, \delta}$ satisfies the recursion $C_{R, \delta}=\sum_{l=\delta-R+1}^{\delta} C_{R-1, l}$ with $C_{0,0}=C_{i, 0}=1$ for $i=1, \ldots, R$.

We can relate this to Kendall's correlation. The Kendall correlation between the utility and $f(\cdot)$ for all possible sequences is given by $\tau=(T-2 \delta) / T$, This allows us to consider the probability of observing the sequence with highest expected utility in the $M$ optimal sequences from $f(\cdot)$ directly from the Kendall correlation using the result above.

If the Kendall correlation is strong between the expected utility and the surrogate function, then we can simplify the result further. In particular, if $\delta \leq R-1$, then $N_{R, \delta}=C_{R-1, \delta}$. This leads to the following corollary.

Corollary 1. If $\delta \leq R-1$ then the sequence of tasks with highest expected utility will be in the $M$ sequences with highest value of the surrogate function with probability

$$
\sum_{m=1}^{M} \frac{C_{R-2, \delta-m+1}}{C_{R-1, \delta}}
$$

This will be the case if $\tau \geq 1-\frac{2}{T}(R-1)$. We can use the result from the corollary to provide 
us with a value for $M$ which guarantees that we find the optimal sequence, provided we know $\delta$. The proof is given in the Supplementary Material.

Proposition 2. If $\delta \leq R-1$, then if we choose $M=\delta+1$ the optimal sequence will be in the $M$ sequences with the highest value of $f(\cdot)$ with probability 1 . If $M=\delta$, this probability is

$$
1-\frac{1}{C_{R-1, \delta}}
$$

When the number of items to sequence is large, the value of $M$ needed to guarantee the optimal sequence will be large. Although we can estimate the value of $\delta$ from the training set of sequences, we do not know its population value and so, while these results can give us a feel for a suitable value of $M$, they are not sufficient to allow us to choose $M$. In Section 5 we provide guidance on choosing the values of $N$ and $M$ based on an extensive simulation study.

We can illustrate the results using a simple example, which is given in the Supplementary Material.

\section{Sequencing reliability growth tasks}

\subsection{Reliability of hardware products under development}

We consider the model developed in Johnston et al. [2006], Quigley and Walls [2006], which is adapted from IEC 61014 and explicates the relationship between the reliability of a system under development and planned development activities. The model is predicated on the concept of concerns, which are possible faults within a system, which may then lead to system failure. The following provides definitions to four key concepts in the model.

Definition 1. Reliability is the ability of a system to perform a required function under stated conditions for a stated period of time.

Definition 2. A failure is the inability of a system to perform a required function under stated conditions for a stated period of time.

Definition 3. A fault is an imperfection or deficiency in a system such that the system will fail, i.e. not perform a required function under stated conditions for a stated period of time.

Definition 4. A concern is a possible fault, i.e. imperfection or deficiency in a system such that the system will fail. 
The reliability of the system is assessed by a probability distribution, which is developed by identifying a set of concerns, assessing the probability that each concern is a fault and specifying distributions describing the time each fault will be realised as a failure. The parameters can be assessed using expert judgement elicitation or historical data [Quigley and Walls, 1999, Walls and Quigley, 2001].

Suppose the concerns associated with the current system design are labelled $i=1, \ldots, I$. Let us define $Z_{i}$ to be an indicator variable such that $Z_{i}=1$ if concern $i$ is a fault and 0 otherwise. Define $R_{i}(t)$ to be the probability that concern $i$ would result in failure after time $t$ conditional on concern $i$ being a fault. If the realisations of failures are independent then the reliability of the system can be expressed as

$$
R(t, \boldsymbol{z})=\prod_{i=1}^{I}\left[1-z_{i}\left(1-R_{i}(t)\right)\right],
$$

where $Z$ is the vector of indicator variables for all concerns. For each concern we have an associated probability of the concern being a fault, elicited from expert judgement elicitation, $\lambda_{i}=\operatorname{Pr}\left(Z_{i}=\right.$ 1 ), so the expectation of $R(t, \boldsymbol{z})$ with respect to $\boldsymbol{Z}$ is easily obtained by substituting $z_{i}$ for $\lambda_{i}$, under the assumption that $Z_{i} \Perp Z_{i^{\prime}}$, for $i \neq i^{\prime}$.

During a reliability development program activities are performed assessing the design of the system, where the outcome of each activity is either to confirm a concern as a fault or provide evidence to the contrary. We assume that once a fault has been confirmed it is designed out of the system. The reliability growth during the program is captured through Bayesian updating of $\lambda_{i}$ given test data. We define the following two indicator variables. Denote $\kappa_{j}$ to represent whether activity $j$, for $j=1, \ldots, J$, has been conducted or not, i.e. $\kappa_{j}=1$ or 0 respectively. Let $D_{i, j}$ indicate whether concern $i$ is realised as a fault in activity $j\left(D_{i, j}=1\right)$, or not $\left(D_{i, j}=0\right)$.

We define $p_{i, j}$ as the conditional probability associated with $D_{i, j}, p_{i, j}=\operatorname{Pr}\left(D_{i, j}=1 \mid Z_{i}=1\right)$, and this is is specified through expert elicitation based on the idea of an efficacy matrix [Johnston et al., 2006, Wilson and Quigley, 2016].

It is sufficient for the model to utilise the indicator $D_{i}=\max _{j=1, \ldots, J}\left(\kappa_{j} D_{i, j}\right)$. Assuming that test outcomes are independent, the updated probability of a concern being a fault given a test 
program has not realised the concern as a fault, through Bayes Theorem, is

$$
\operatorname{Pr}\left(Z_{i}=z_{i} \mid D_{i}=0\right)=\left\{\begin{array}{l}
\frac{1-\lambda_{i}}{1-\lambda_{i}\left[1-\prod_{j=1}^{J}\left(1-p_{i, j}\right)^{\kappa_{j}}\right]}, z_{i}=0, \\
\frac{\lambda_{i} \prod_{j=1}^{J}\left(1-p_{i, j}\right)^{\kappa_{j}}}{1-\lambda_{i}\left[1-\prod_{j=1}^{J}\left(1-p_{i, j}\right)^{\kappa_{j}}\right]}, z_{i}=1
\end{array}\right.
$$

with $\operatorname{Pr}\left(Z_{i}=1 \mid D_{i}=1\right)=1$ and $\operatorname{Pr}\left(Z_{i}=0 \mid D_{i}=1\right)=0$ as, when an item fails due to concern $i$, it must be a fault.

This allows us to evaluate the prior expectation of the reliability, given a planned set of development activities. The reliability in this case can be expressed as $R(t, \boldsymbol{z})=\prod_{i=1}^{I} R_{i}(t)^{I\left[z_{i}>d_{i}\right]}$, where $I\left[z_{i}>d_{i}\right]$ is an indicator function which takes the value 1 if $z_{i}>d_{i}$ and 0 otherwise, under the assumption that once a fault has been identified in a task it is designed out of the system with probability one.

$$
\mathrm{E}_{\boldsymbol{D}}\left\{\mathrm{E}_{\boldsymbol{Z} \mid \boldsymbol{D}}[R(t, \boldsymbol{z})]\right\}=\prod_{i=1}^{I}\left[1-\left(1-R_{i}(t)\right) \lambda_{i} \prod_{j=1}^{J}\left(1-p_{i, j}\right)^{\kappa_{j}}\right]
$$

where $\boldsymbol{D}$ is the vector of indicator variables $D_{i}$. A full derivation of this result is given in the Supplementary Material.

\subsection{Optimal sequencing of reliability growth tasks}

We could perform all of the possible reliability growth tasks for a system. Following this, if the system meets some pre-specified reliability target $R_{0}$, it will be released. However, if we were to reach our reliability target after fewer than the allocated tasks, then we would stop testing and save time and money. Therefore, finding the optimal sequence of reliability tasks is an important question.

We need to consider the probability distribution of the reliability, as we are interested in quantities of the form $\operatorname{Pr}\left(R(t, \boldsymbol{z}) \geq R_{0}\right)=\alpha(t)$. We transform the reliability so that it is not restricted to $[0,1]$. Specifically,

$$
g(t, \boldsymbol{z})=\log [R(t, \boldsymbol{z})]
$$

Assume that the transformed reliability follows a Normal distribution $g(t, \boldsymbol{z}) \sim N(m(t), v(t))$. Justification for this choice will be given later. Then the probabilities of interest are $\operatorname{Pr}(R(t, \boldsymbol{z}) \geq$ 
$\left.R_{0}\right)=\operatorname{Pr}\left(g(t, \boldsymbol{z}) \geq \log R_{0}\right)$.

We can fully specify the distribution of the reliability by specifying $m(t), v(t)$ as $m(t)=$ $\mathrm{E}_{\boldsymbol{D}}\left\{\mathrm{E}_{\boldsymbol{Z} \mid \boldsymbol{D}}[g(t, \boldsymbol{z})]\right\}$ and $v(t)=\mathrm{E}_{\boldsymbol{D}}\left\{\mathrm{E}_{\boldsymbol{Z} \mid \boldsymbol{D}}\left[g(t, \boldsymbol{z})^{2}\right]\right\}-\mathrm{E}_{\boldsymbol{D}}\left\{\mathrm{E}_{\boldsymbol{Z} \mid \boldsymbol{D}}[g(t, \boldsymbol{z})]\right\}^{2}$.

These are not fast and efficient calculations to perform. Each vector $\boldsymbol{d}$ and $\boldsymbol{z}$ are of length $I$ with each element having two possible states, 0 and 1 . The number of sequences of length $J$ is $J$ !. Therefore the total number of calculations which would be necessary to evaluate the probability distribution of $g(t, \boldsymbol{z})$ for all sequences of length $J$ is $G=2^{2 I+1} \times J$ !. For example, if $I=5, J=5$ then $G=245,760$ and if $I=15, J=14$ (still reasonably small) then $G=1.87 \times 10^{20}$. In practice, for a reasonably large problem, it is not going to be possible to evaluate all of the required expectations exactly.

We can approximate the logarithm of the reliability by taking a rare event approximation to obtain

$$
-\log [R(t, \boldsymbol{z})]=\sum_{i=1}^{I}\left(1-R_{i}(t)\right) z_{i}-\left[\sum_{i=1}^{I}\left(1-R_{i}(t)\right) z_{i} \sum_{j=1}^{J} \kappa_{j}\left(1-\mu_{i, j}\right)\right]
$$

where $\mu_{i, j}$ is 1 if task $j$ finds fault $i$ given that fault $i$ exists and 0 if it does not find $i$ when it exists. In this case,

$$
\begin{aligned}
m(t) & =-\left[\sum_{i=1}^{I}\left(1-R_{i}(t)\right) \lambda_{i}-\left(\sum_{i=1}^{I}\left(1-R_{i}(t)\right) \lambda_{i} \sum_{j=1}^{J} \kappa_{j}\left(1-p_{i, j}\right)\right)\right] \\
v(t) & =\sum_{i=1}^{I}\left[\left(1-R_{i}(t)\right) \lambda_{i}\right]^{2} \sum_{j=1}^{J} \kappa_{j}\left(1-p_{i, j}\right) p_{i, j} .
\end{aligned}
$$

By the Lyapanov Central Limit Theorem [Knight, 2000] the distribution of the approximation is asymptotically Normal with increasing numbers of activities.

Using the approximations the total number of calculations required to solve the design problem for a sequence of length $J$ reduces to $H=2 I J \times J$ !. In the specific cases given above, if $I=$ $5, J=5$ then $H=6000$ and if $I=15, J=14$ then $H=3.66 \times 10^{13}$. We see that the number of calculations required has been significantly reduced.

\subsection{Bayesian expected utility solution}

We define a utility function which incorporates the uncertainty on the reliability $R(t, \boldsymbol{z})$. The multiattribute utility function we define will include this uncertainty in both the conditional utilities for 
financial cost and time cost. Thus the multi-attribute utility function will depend on attributes $(Y, \chi)$, where $Y$ represents financial cost and $\chi$ represents time.

Recall that the possible sequences of tasks of length $J$ are given by $\boldsymbol{x}=\left(x_{1}, \ldots, x_{J}\right)$ and that $\boldsymbol{x} \in \mathcal{S}^{J}$, where $\mathcal{S}^{J}$ represents the set of all of the permutations of sequences of length $J$. Then the Bayesian optimal sequence of tasks is given by

$$
\operatorname{argmax}_{\boldsymbol{x} \in \mathcal{S}^{J}}\left[\mathrm{E}_{\boldsymbol{D}}\left\{\mathrm{E}_{\boldsymbol{Z} \mid \boldsymbol{D}}[U(\boldsymbol{x}, \boldsymbol{z})]\right\}\right]
$$

where the utility function $U(\boldsymbol{x}, \boldsymbol{z})$ incorporates the probability distribution of $R(t, \boldsymbol{z})$ in the following way.

Suppose that, for sequence $\boldsymbol{x}$, the costs associated with the individual tasks are $\left(y\left(x_{1}\right), \ldots, y\left(x_{J}\right)\right)$ and the times are $\left(\chi\left(x_{1}\right), \ldots, \chi\left(x_{J}\right)\right)$. Then if we perform $j$ tasks the total costs and times will be given by $y_{j}^{(t o t)}(\boldsymbol{x})=\sum_{k=1}^{j} y\left(x_{k}\right)$ and $\chi_{j}^{(t o t)}(\boldsymbol{x})=\sum_{k=1}^{j} \chi\left(x_{k}\right)$ respectively. Introducing the rule that we stop testing if we reach the target reliability, the cost and time for sequence $\boldsymbol{x}$ are

$$
C(\boldsymbol{x}, \boldsymbol{z})=\sum_{j=1}^{J}\left[y_{j}^{(t o t)}(\boldsymbol{x}) \gamma_{j} \prod_{k=1}^{j-1}\left(1-\gamma_{j}\right)\right], T(\boldsymbol{x}, \boldsymbol{z})=\sum_{j=1}^{J}\left[\chi_{j}^{(t o t)}(\boldsymbol{x}) \gamma_{j} \prod_{k=1}^{j-1}\left(1-\gamma_{j}\right)\right]
$$

where $\gamma_{j}$ is an indicator variable which takes the value 1 if $R(t, \boldsymbol{z})>R_{0}$ and 0 if not. If we assume utility independence between cost and time we can then define the general utility function to be a binary node:

$$
U(\boldsymbol{x}, \boldsymbol{z})=q_{1} U(C(\boldsymbol{x}, \boldsymbol{z}))+q_{2} U(T(\boldsymbol{x}, \boldsymbol{z}))+q_{3} U(C(\boldsymbol{x}, \boldsymbol{z})) U(T(\boldsymbol{x}, \boldsymbol{z}))
$$

for trade-off parameters $q_{1} \geq 0, q_{2} \geq 0$ and $-q_{i} \leq q_{3} \leq 1-q_{i}$ for $i=1,2$ such that $q_{1}+q_{2}+q_{3}=1$. Examples of suitable risk averse marginal utility functions for financial cost and time would be

$$
U(C(\boldsymbol{x}, \boldsymbol{z}))=1-\left(C(\boldsymbol{x}, \boldsymbol{z}) / Y_{0}\right)^{2}, U(T(\boldsymbol{x}, \boldsymbol{z}))=1-\left(T(\boldsymbol{x}, \boldsymbol{z}) / \chi_{0}\right)^{2},
$$

where $Y_{0}$ is the maximum budget and $\chi_{0}$ is the maximum time to carry out the tasks, however any suitable functions on $[0,1]$ could be used.

We see that, to calculate the expected utility of a particular sequence, we need $\mathrm{E}\left[\gamma_{j}\right]$, for all $j$, which are the $\operatorname{Pr}\left(R(t, \boldsymbol{z})>R_{0}\right)$ for a particular stage of a specific sequence.

Although the rare event approximation reduces the number of calculations required to evaluate 
the probability distribution of the reliability, and hence the expected utilities of the sequences of tasks, even for a small problem with $I=15, J=14$ the number of calculations required to solve the decision problem is not feasible to undertake in practice. Instead, we propose to use the surrogate functions developed in Section 2 to approximate the expected utilities and solve the decision problem approximately. This approach is illustrated using an example and evaluated using a simulation study in the next two sections.

\section{Illustrative example}

\subsection{Background}

Suppose that in an elicitation engineers identify 15 concerns in a product under development. There are 9 possible tasks which the engineers could carry out to identify if these concerns are faults and, if they are, design them out. This means that in all there are 362,880 possible sequences of the tasks which could be carried out.

Each task has associated with it a cost of between 0 and 50 units and a duration of between 0 and 20 units. The target reliability, which would be assessed by the decision maker, is 0.8 , the maximum time is 150 units and the maximum total cost is 132 units. If the conditional rate of failure for concern $i$, given that it is a fault, can be thought of as constant over time a suitable choice for the time to failure is the exponential distribution. This results in a reliability function of $R_{i}(t)=\exp \left\{-\epsilon_{i} t\right\}$, where $\epsilon_{i}$ is the rate of failures resulting from concern $i$, given that it is a fault. The final parameters which need to be specified are the trade-off parameters for the binary utility function. In the example, the $\lambda_{i}$ are between 0 and 0.5 , approximately $50 \%$ of the $p_{i, j}$ are equal to zero indicating that task $j$ will not find concern $i$, given that it is a fault, and the rest are between 0 and 0.5 and each $\epsilon_{i}$ is chosen to be 0.02 . The values of $\lambda_{i}$ and $p_{i, j}$ used in the example are given in Table 2.

\subsection{Emulation}

We wish to sequence the tasks in order to minimise the expected cost and time. We do so by maximising the prior expected utility as outlined above. We have a single binary utility function for cost and time. The conditional utilities used are those in (2). Suppose that initially the decision maker felt that the utility of each was equally important. Then $q_{1}=1 / 2, q_{2}=1 / 2, q_{3}=0$.

For this illustrative example with $J=9$, evaluating the expected utilities for all of the sequences 


\begin{tabular}{|c|c|ccccccccc|}
\hline$i$ & $\lambda_{i}$ & $p_{i, 1}$ & $p_{i, 2}$ & $p_{i, 3}$ & $p_{i, 4}$ & $p_{i, 5}$ & $p_{i, 6}$ & $p_{i, 7}$ & $p_{i, 8}$ & $p_{i, 9}$ \\
\hline 1 & 0.13 & 0.00 & 0.19 & 0.47 & 0.00 & 0.00 & 0.00 & 0.00 & 0.00 & 0.25 \\
2 & 0.19 & 0.00 & 0.40 & 0.00 & 0.00 & 0.39 & 0.26 & 0.01 & 0.00 & 0.24 \\
3 & 0.29 & 0.22 & 0.00 & 0.00 & 0.33 & 0.00 & 0.00 & 0.00 & 0.00 & 0.04 \\
4 & 0.45 & 0.17 & 0.17 & 0.00 & 0.43 & 0.00 & 0.22 & 0.20 & 0.00 & 0.00 \\
5 & 0.10 & 0.00 & 0.00 & 0.00 & 0.39 & 0.23 & 0.00 & 0.33 & 0.00 & 0.32 \\
6 & 0.45 & 0.00 & 0.00 & 0.49 & 0.18 & 0.00 & 0.00 & 0.00 & 0.50 & 0.00 \\
7 & 0.47 & 0.00 & 0.00 & 0.00 & 0.29 & 0.00 & 0.46 & 0.28 & 0.49 & 0.34 \\
8 & 0.33 & 0.12 & 0.00 & 0.00 & 0.05 & 0.31 & 0.16 & 0.00 & 0.00 & 0.00 \\
9 & 0.31 & 0.00 & 0.22 & 0.44 & 0.00 & 0.00 & 0.00 & 0.43 & 0.00 & 0.32 \\
10 & 0.03 & 0.30 & 0.15 & 0.00 & 0.44 & 0.00 & 0.47 & 0.36 & 0.00 & 0.14 \\
11 & 0.10 & 0.06 & 0.16 & 0.13 & 0.00 & 0.00 & 0.28 & 0.00 & 0.48 & 0.00 \\
12 & 0.09 & 0.41 & 0.00 & 0.48 & 0.00 & 0.00 & 0.46 & 0.13 & 0.00 & 0.17 \\
13 & 0.34 & 0.00 & 0.20 & 0.34 & 0.21 & 0.00 & 0.46 & 0.00 & 0.00 & 0.00 \\
14 & 0.19 & 0.38 & 0.24 & 0.24 & 0.00 & 0.00 & 0.26 & 0.00 & 0.35 & 0.00 \\
15 & 0.38 & 0.31 & 0.00 & 0.00 & 0.00 & 0.00 & 0.00 & 0.01 & 0.44 & 0.00 \\
\hline
\end{tabular}

Table 2: The values of $\lambda_{i}$ and $p_{i, j}$ used in the example.

takes 102 seconds in R version 3.3.1 on a machine with 16GB of RAM and an Intel Core i76700@3.40 GHz processor. If we increase the number of tasks this exhaustive search would take around 17 minutes for $J=10,3.1$ hours for $J=11,37$ hours for $J=12,20$ days for $J=13$, 284 days for $J=14$ and 11.7 years for $J=15$. Thus, while we have chosen a number of tasks for which we can evaluate the success of the emulator by comparison with the exact solution in this case, for moderately larger decision problems we would be unable to solve the problem exactly.

We suppose that we have a budget of $B=100$ evaluations of the expected utility, which will be split between $N$ evaluations at the training sample and $M$ evaluations based on the top $M$ sequences under the emulator. In order to focus attention on the ability of the emulator, rather than the choice of training sample, we choose the simplest possible design for our training sample, i.e. $N$ sequences sampled uniformly from the set of all $J$ ! sequences. With such a design, the empirical results of Section 5 suggest that the optimal split of our budget is approximately $N=60$ and $M=40$, giving roughly a $75 \%$ chance of obtaining the optimal sequence. We use the Pearson correlation as our measure of correlation $c(\psi)$, again due to its superior empirical performance; see Section 5 . We first fit the initial surrogate based on the Benter model, $\hat{f}(\cdot)$, as described in Section 2.1. The optimal choice for this illustrative example is $\hat{\boldsymbol{\theta}}=(1.00,0.79,2.36,2.72,0.38,3.20,0.99,3.45,0.89)$ and $\hat{\boldsymbol{\alpha}}=(1.37,1.43,1.58,1.31,0.57,0.18,0.03,0.13,0)$. This gives a sample Pearson correlation coefficient of $c(\hat{\psi})=0.984$, suggesting that we may be able to use $\hat{f}(\cdot)$ as a surrogate for the logit expected utility in a search for the optimal sequence. It is good practice to produce a visual check 
of the association between the output under the surrogate and the logit expected utilities (to decide if the association is likely to produce a good emulator). The values of the logit expected utility and the surrogate function values at the 60 training points for this choice of $\psi$ are given in the left hand side of Figure 1 and show a good correspondence.
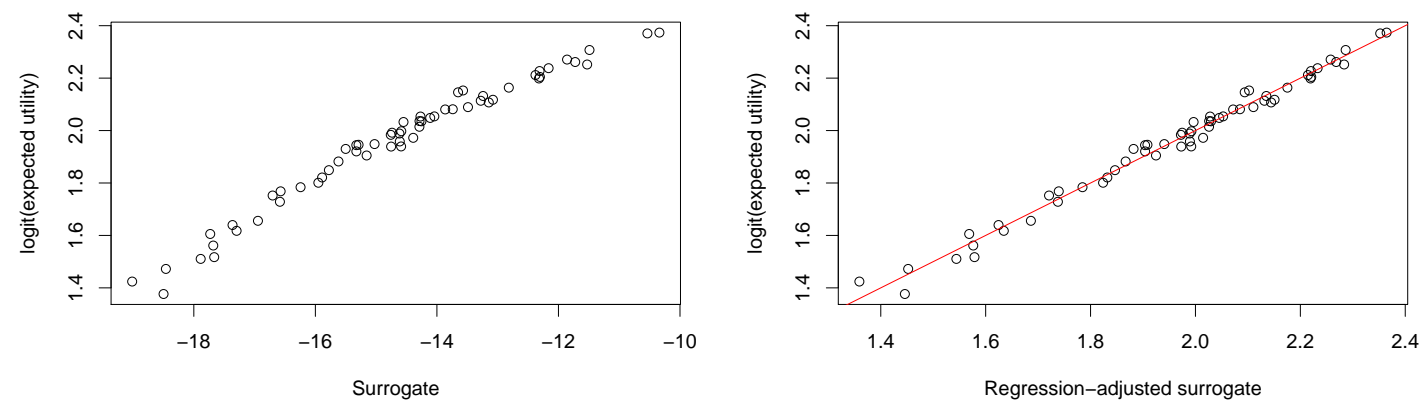

Figure 1: Logit transformed expected utilities versus surrogate function values for the training sample of sequences under the initial surrogate $\hat{f}(\cdot)$ (left) and the regression-adjusted surrogate $f^{\star}(\cdot)$ (right).

Whilst the estimated parameters are largely a vehicle for obtaining an emulator, they do provide some insight into the solution of the design problem. The rank order of the $\hat{\theta}_{k}$ from largest to smallest should be similar to the order of tasks with the highest value of the surrogate function: in this case the order is $(8,6,4,3,1,7,9,2,5)$, suggesting task 8 is performed first, followed by task 6 and so on. The relative values of the $\hat{\theta}_{k}$ also provide a rough guide to how persistent the tasks are likely to be in the sequences with the highest values of the surrogate function; for example we can see that $\hat{\theta}_{5}$ is much lower than the other parameters and so task 5 is likely to always be scheduled last in the sequences with high values of the surrogate function. Similarly, $\hat{\alpha}_{j}$ can be interpreted as the importance of the task that is performed $j$ th in the sequence. We see that the values for $\alpha_{1}$ to $\alpha_{4}$ (and possibly $\alpha_{5}$ ) are all relatively large and then the values start to tail off. This suggests that the first four (or five) tasks may be the important tasks to schedule, perhaps due to the reliability target being reached by then, with the last few not as important (in which case the order in which the tasks are performed may have little impact on the expected utility).

We can evaluate the suitability of the regression-adjusted surrogate function; see Section 2.1.1. In this case we obtain $\widehat{\boldsymbol{\beta}}=(2.31,-0.07,-0.01,0.00)$ and this gives a sample correlation coefficient of 0.993. The logit expected utilities are plotted against the regression-adjusted surrogate function values in the right hand side of Figure 1. The correlation is very high for both surrogate 
functions, indicating that either would be a good emulator, however, in what follows we use the regression-adjusted surrogate as that gave the higher Pearson correlation.

We evaluate the regression-adjusted surrogate function at all 9! sequences of tasks; this takes approximately 17 seconds which is 6 times quicker than evaluating all of the expected utilities. For increasing numbers of tasks the emulation would take around 3 minutes for $J=10,31$ minutes for $J=11,6$ hours 15 minutes for $J=12,3.4$ days for $J=13,47$ days for $J=14$ and 1.9 years for $J=15$. Each of these numbers represents a significant speed up over the exhaustive enumeration of the expected utility. However, even for moderate $J$ complete enumeration may be infeasible. This potential drawback is easily addressed by the probabilistic nature of the surrogate model, as described in Section 5.4.

Figure 2 plots the logit transformed expected utility against the regression-adjusted surrogate function for all 362,880 sequences.

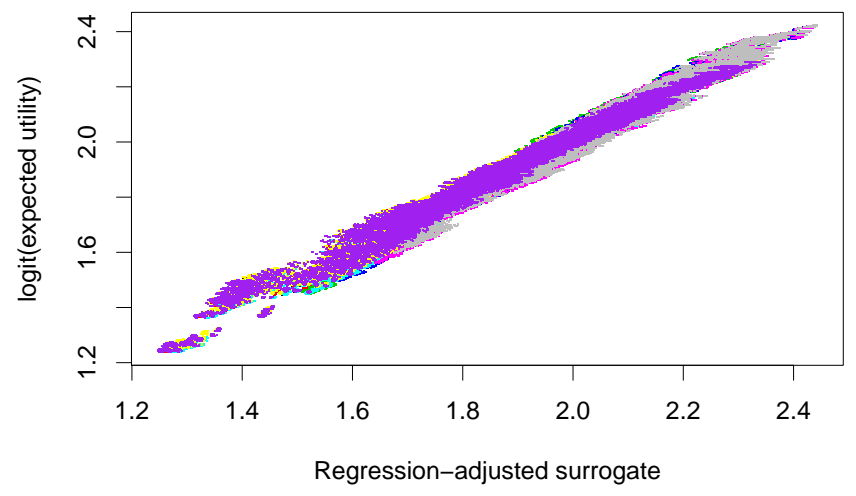

Figure 2: Logit transformed expected utilities versus regression-adjusted surrogate function values for all sequences $\boldsymbol{x}$; the colours indicate the first task in that sequence (1=black, $2=$ red, $3=$ green, 4=blue, 5=cyan, $6=$ magenta, $7=$ yellow, $8=$ grey, 9=purple).

The points in Figure 2 are colour-coded by the first task in each sequence. There is a clear positive relationship between the expected utilities and the surrogate output. The Pearson correlation coefficient is 0.990 . As there is not perfect correspondence between $U(\boldsymbol{x})$ and $f(\boldsymbol{x})$, we propose the remaining $B-N=M=40$ candidate sequences which have the highest values under the emulator $f^{\star}(\cdot)$ and then evaluate the expected utility at these $M$ candidate values. In the interests of space, only the top ten sequences in terms of their values of the surrogate function are given in Table 3. Whilst the sequence $\boldsymbol{x}=(8,6,4,3,1,7,2,9,5)$ has the largest value under the emulator it does 


\begin{tabular}{|c|c|c|c|c|c|c|c|c|c|c|c|c|}
\hline Sequence $(m)$ & \multicolumn{9}{|c|}{ Task sequence } & $f^{\star}(\boldsymbol{x})$ & $\operatorname{logit} U(\boldsymbol{x})$ & $\hat{P}$ \\
\hline 1 & 8 & 6 & 4 & 3 & 1 & 7 & 2 & 9 & 5 & 2.442344 & 2.422417 & 0.17 \\
\hline 2 & 8 & 6 & 4 & 3 & 1 & 7 & 9 & 2 & 5 & 2.442185 & 2.422419 & 0.19 \\
\hline 3 & 8 & 6 & 4 & 3 & 7 & 1 & 2 & 9 & 5 & 2.442108 & 2.422063 & 0.21 \\
\hline 4 & 8 & 6 & 4 & 3 & 7 & 1 & 9 & 2 & 5 & 2.441948 & 2.422065 & 0.23 \\
\hline 5 & 8 & 6 & 4 & 3 & 1 & 9 & 2 & 7 & 5 & 2.441792 & 2.422417 & 0.25 \\
\hline 6 & 8 & 6 & 4 & 3 & 1 & 9 & 7 & 2 & 5 & 2.441504 & 2.422418 & 0.27 \\
\hline 7 & 8 & 6 & 4 & 3 & 7 & 9 & 2 & 1 & 5 & 2.441485 & 2.422040 & 0.29 \\
\hline 8 & 8 & 6 & 4 & 3 & 7 & 9 & 1 & 2 & 5 & 2.441180 & 2.422044 & 0.32 \\
\hline 9 & 6 & 8 & 4 & 3 & 1 & 7 & 2 & 9 & 5 & 2.441053 & 2.422381 & 0.34 \\
\hline 10 & 8 & 6 & 4 & 3 & 1 & 2 & 9 & 7 & 5 & 2.440947 & 2.422380 & 0.36 \\
\hline
\end{tabular}

Table 3: The ten sequences with the highest values of $f^{\star}(\boldsymbol{x})$ and their corresponding logit expected utilities. The final column gives an estimate $\hat{P}$ for the probability that the optimal sequence is to be found in the top $m$ sequences under the emulator for examples of this type.

not have the highest expected utility. Nevertheless, these top 10 sequences all have high expected utility. The sequence which gives the maximum expected utility out of the $B=N+M=100$ at which the expected utility was calculated, and is therefore our putative optimal sequence, is $\tilde{\boldsymbol{x}}=(8,6,4,3,1,7,9,2,5)$ which is ranked 2 nd in terms of the emulator. Also in Table 3 we give an estimate $\hat{P}$ for the probability that the optimal sequence is to be found in the top $m$ sequences under the emulator for examples of this type. These estimates are based on a regression analysis of the simulation results of Section 5 .

As mentioned previously, we can evaluate all of the expected utilities for this example. The sequences ranked by the highest expected utilities are given in Table 4. In practice, these sequences would not typically be known. We show them here to investigate the ability of the surrogate function to identify the globally optimal sequence.

The optimal sequence of tasks, that with the largest expected utility, is $\hat{\boldsymbol{x}}=(8,6,4,3,1,7,9,2,5)$. This matches $\tilde{\boldsymbol{x}}$, the putative optimal sequence which was highlighted in our candidate list. In this illustrative example, we have found the optimal sequence using only $B=100$ evaluations of the expensive expected utility. Our simulation results in Section 5 suggest that in similar examples, with this choice of $N$ and $M$ we would obtain the optimal sequence roughly $75 \%$ of the time. Even when we do not find the optimal sequence in the $B$ evaluations, we still find sequences with close to optimal values of the expected utility. For example, in this illustrative example, the sequence with maximum value of the surrogate function has the 5 th highest expected utility and matches the optimal sequence in the first five performed tasks. So, even if we set $M=1$ we would end up with only a marginally sub-optimal sequence. 


\begin{tabular}{|c|ccccccccc|c|c|}
\hline Sequence & \multicolumn{10}{|c|}{$f^{\dagger}(\boldsymbol{x})$} & $U(\boldsymbol{x})$ \\
\hline 1 & 8 & 6 & 4 & 3 & 1 & 7 & 9 & 2 & 5 & 0.9199880 & 0.9185209 \\
2 & 8 & 6 & 4 & 3 & 1 & 7 & 9 & 5 & 2 & 0.9197039 & 0.9185209 \\
3 & 8 & 6 & 4 & 3 & 1 & 9 & 7 & 2 & 5 & 0.9199380 & 0.9185209 \\
4 & 8 & 6 & 4 & 3 & 1 & 9 & 7 & 5 & 2 & 0.9196522 & 0.9185209 \\
5 & 8 & 6 & 4 & 3 & 1 & 7 & 2 & 9 & 5 & 0.9199998 & 0.9185208 \\
6 & 8 & 6 & 4 & 3 & 1 & 9 & 2 & 7 & 5 & 0.9199592 & 0.9185208 \\
7 & 8 & 6 & 4 & 3 & 1 & 7 & 2 & 5 & 9 & 0.9196663 & 0.9185208 \\
8 & 8 & 6 & 4 & 3 & 1 & 9 & 2 & 5 & 7 & 0.9195836 & 0.9185208 \\
9 & 8 & 6 & 4 & 3 & 1 & 7 & 5 & 9 & 2 & 0.9197978 & 0.9185195 \\
10 & 8 & 6 & 4 & 3 & 1 & 7 & 5 & 2 & 9 & 0.9197485 & 0.9185195 \\
\hline
\end{tabular}

Table 4: The ten sequences with the highest values of $U(\boldsymbol{x})$ and their corresponding values under $f^{\dagger}(\cdot)$.

As illustrated above, in this example we have been able to evaluate the expected utility at all possible sequences of tasks and therefore been able to identify the optimal sequence, but our methodology is designed for scenarios where this is not possible. In such situations, a practitioner will not know whether they have obtained the optimal sequence, or whether their putative optimal sequence is close to the optimal. We therefore recommend a simple graphical diagnostic plot along the lines of that in Figure 3. This compares graphically the logit transformed expected utilities in
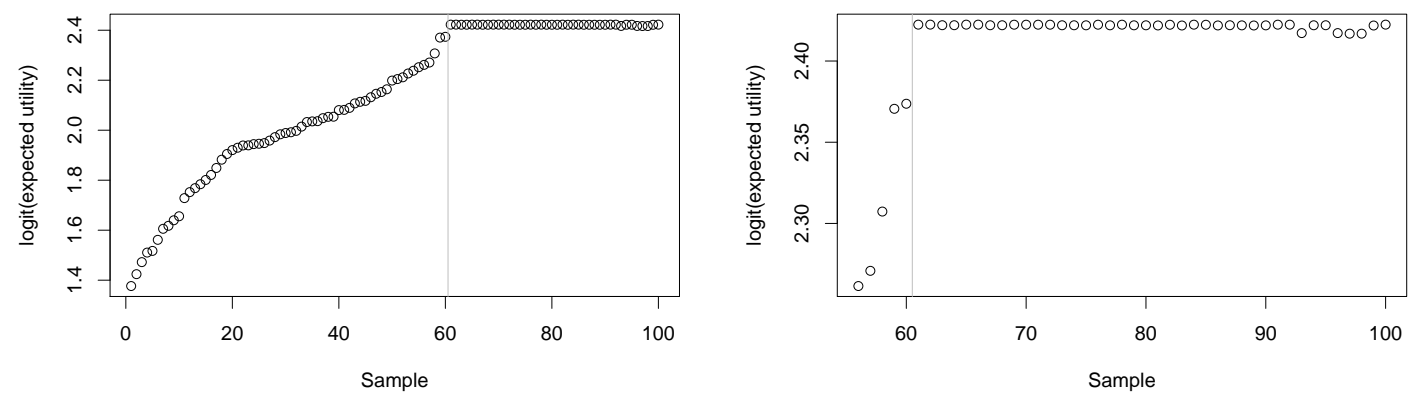

Figure 3: Logit transformed expected utilities versus sample number. In each plot the points to the left of the vertical line represent the training samples (ordered by increasing expected utility) and the points to the right of the line correspond to the $M$ sequences suggested by the emulator in decreasing order of the regression-adjusted surrogate function value. The left-hand plot shows all $B=100$ samples. The right-hand plot focuses on the top 5 from the training sample and the $M$ sequences proposed from the emulator.

the training sample of size $N$ with those in the evaluation sample of size $M$. From such a plot, a practitioner can see the extent to which the $M$ emulator-proposed sequences all have much larger 
expected utilities than those in the training sample and also the extent to which the expected utilities of the $M$ emulator-proposed sequences are all very similar. This is reassuring as it suggests that the putative optimal sequence is probably close to optimal (in terms of the overall variation in logit expected utilities that are observed in the training sample). So, whilst, in a real example, there are no guarantees that the optimal sequence has been found, such a graphical diagnostic can provide some reassurance that the putative optimal sequence is satisfactory.

As suggested by one of the referees, an alternative strategy for checking the stability of the emulator and of the putative optimal sequence is to split the training sample into sub-samples and perform the emulator fitting/exploration process on each sub-sample. Our experience, as reported in the Supplementary Material, is that not much insight is gained by this splitting of the training sample and so we have chosen not to pursue that procedure here.

\subsection{Results}

The optimal ordering of tasks has an expected utility of 0.919 and corresponds to the sequence $(8,6,4,3,1,7,9,2,5)$. That is, we first carry out task 8 and then if we haven't met the reliability target we move on to task 6 . If we still haven't met the reliability target we then carry out task 4 , etc.

We consider the costs, times and probabilities of reaching the target reliability following each task. They are given in Table 5.

\begin{tabular}{|c|ccccccccc|}
\hline Task & 8 & 6 & 4 & 3 & 1 & 7 & 9 & 2 & 5 \\
\hline Cost & 7 & 23 & 31 & 37 & 48 & 60 & 66 & 115 & 132 \\
Time & 10 & 24 & 26 & 28 & 29 & 48 & 61 & 63 & 73 \\
Probability & 0.00 & 0.08 & 0.48 & 0.87 & 0.98 & 0.99 & 1.00 & 1.00 & 1.00 \\
\hline
\end{tabular}

Table 5: Various quantities of interest broken down by task in the optimal sequence.

From the table we see that it is not likely that we will have to perform all of the tasks to achieve the target reliability. It is likely that only 4 or 5 tasks will be needed and that we can reach the reliability target with a spend of 37-48 and a time of 28-29. Note that this coincides with our interpretation of the $\alpha$ parameters; the first 4 or 5 tasks have relatively large values of $\alpha$ compared to the other tasks. We see that we are likely to spend a larger proportion of our total time than our total budget for testing. 


\section{Simulation studies}

\subsection{Choice of surrogate model and correlation function}

A simulation study was performed to assess the performance of several surrogate models based on parametric probabilistic models for permutations: the Benter model (B) as described in Section 2.1; the Plackett-Luce model (PL) which is obtained from the Benter model by setting $\boldsymbol{\alpha}=\mathbf{1}$; and the reverse Plackett-Luce model (RPL) which is obtained via the PL model evaluated at the reverse sequence of tasks. We did not include the regression-adjustment in the simulation study since it can be applied to any surrogate function and will improve upon it. A range of correlation functions (Pearson, Spearman and Kendall) were considered as the objective function for the fitting procedure for each of the putative surrogate models. We have considered numbers of tasks $J$ ranging from 6 to 10 and three sets of trade-off parameters: (a) $q_{1}=1 / 2, q_{2}=1 / 2, q_{3}=0$, (b) $q_{1}=1 / 3, q_{2}=$ $1 / 3, q_{3}=1 / 3$ and (c) $q_{1}=2 / 3, q_{2}=2 / 3, q_{3}=-1 / 3$. The problem set-up follows that in Section 4 in which we set $\epsilon_{i}=\epsilon=0.02, t=100, R_{0}=0.8, T_{0}=90$. To focus attention on the performance of the emulator, rather than the effect that the design of the training samples has on performance, we use the randomly sampled design strategy that was used for the illustrative example in Section 4. In this way we separate the potential capability of each emulator from the issue of training sample design.

For each combination of number of tasks $J$, trade-off parameters $\boldsymbol{q}$ and initial random sample size $N, 100$ sets of expected utilities for all $J$ ! sequences were generated. For each of these 100 simulations we fitted each of the nine combinations of surrogate model and correlation function based on the initial sample of $N$ sequences. We evaluated the surrogate function at all $J$ ! possible sequences.

We focus on the results for $J=9$ tasks and trade-off parameters (b); the differences for the other combinations of trade-off parameters were minimal and other values of $J$ from 6 to 10 gave broadly similar results, though the differences between the performance of the surrogate models and correlation functions increase as $J$ increases.

Table 6 shows the estimated probability that the optimal sequence in terms of expected utility is contained in either the $N$ initial sequences or the further $M$ sequences based on the best $M$ sequences in terms of the surrogate function, for a range of values of $N$ and $M$.

The results in Table 6 show that using Pearson correlation for the objective function is uniformly 


\begin{tabular}{|c|c|c|c|c|c|c|c|}
\hline \multirow[b]{2}{*}{$N$} & \multirow[b]{2}{*}{ Correlation } & \multirow[b]{2}{*}{ Model } & \multicolumn{5}{|c|}{$M$} \\
\hline & & & 10 & 20 & 50 & 100 & 200 \\
\hline \multirow[t]{9}{*}{25} & Pearson & PL & 0.05 & 0.06 & 0.10 & 0.19 & 0.27 \\
\hline & & RPL & 0.09 & 0.15 & 0.25 & 0.33 & 0.50 \\
\hline & & B & 0.19 & 0.30 & 0.46 & 0.60 & 0.73 \\
\hline & Spearman & PL & 0.04 & 0.05 & 0.05 & 0.05 & 0.06 \\
\hline & & RPL & 0.01 & 0.02 & 0.06 & 0.10 & 0.14 \\
\hline & & B & 0.00 & 0.00 & 0.01 & 0.01 & 0.01 \\
\hline & Kendall & PL & 0.01 & 0.01 & 0.02 & 0.03 & 0.06 \\
\hline & & RPL & 0.01 & 0.03 & 0.04 & 0.07 & 0.11 \\
\hline & & B & 0.00 & 0.00 & 0.00 & 0.00 & 0.01 \\
\hline \multirow[t]{9}{*}{50} & Pearson & PL & 0.11 & 0.19 & 0.35 & 0.54 & 0.70 \\
\hline & & RPL & 0.26 & 0.38 & 0.56 & 0.68 & 0.80 \\
\hline & & B & 0.40 & 0.51 & 0.73 & 0.85 & 0.95 \\
\hline & Spearman & PL & 0.02 & 0.04 & 0.06 & 0.17 & 0.27 \\
\hline & & RPL & 0.05 & 0.09 & 0.17 & 0.22 & 0.29 \\
\hline & & B & 0.03 & 0.07 & 0.13 & 0.23 & 0.26 \\
\hline & Kendall & PL & 0.05 & 0.08 & 0.12 & 0.16 & 0.28 \\
\hline & & RPL & 0.06 & 0.18 & 0.22 & 0.28 & 0.35 \\
\hline & & B & 0.06 & 0.10 & 0.13 & 0.19 & 0.25 \\
\hline \multirow[t]{9}{*}{75} & Pearson & PL & 0.15 & 0.29 & 0.49 & 0.67 & 0.77 \\
\hline & & RPL & 0.35 & 0.46 & 0.68 & 0.77 & 0.89 \\
\hline & & B & 0.49 & 0.66 & 0.87 & 0.96 & 0.99 \\
\hline & Spearman & PL & 0.06 & 0.11 & 0.17 & 0.26 & 0.35 \\
\hline & & RPL & 0.16 & 0.23 & 0.29 & 0.43 & 0.56 \\
\hline & & B & 0.17 & 0.21 & 0.32 & 0.42 & 0.54 \\
\hline & Kendall & PL & 0.04 & 0.09 & 0.19 & 0.26 & 0.34 \\
\hline & & RPL & 0.10 & 0.19 & 0.31 & 0.42 & 0.50 \\
\hline & & B & 0.10 & 0.20 & 0.32 & 0.38 & 0.47 \\
\hline \multirow[t]{9}{*}{100} & Pearson & PL & 0.15 & 0.34 & 0.48 & 0.67 & 0.83 \\
\hline & & RPL & 0.37 & 0.59 & 0.81 & 0.92 & 0.98 \\
\hline & & B & 0.54 & 0.72 & 0.94 & 0.98 & 1.00 \\
\hline & Spearman & PL & 0.11 & 0.15 & 0.24 & 0.34 & 0.41 \\
\hline & & RPL & 0.20 & 0.28 & 0.46 & 0.59 & 0.69 \\
\hline & & B & 0.21 & 0.33 & 0.50 & 0.61 & 0.68 \\
\hline & Kendall & PL & 0.07 & 0.13 & 0.24 & 0.31 & 0.39 \\
\hline & & RPL & 0.14 & 0.21 & 0.34 & 0.43 & 0.60 \\
\hline & & B & 0.20 & 0.28 & 0.43 & 0.55 & 0.68 \\
\hline \multirow[t]{9}{*}{200} & Pearson & PL & 0.36 & 0.57 & 0.72 & 0.87 & 0.96 \\
\hline & & RPL & 0.62 & 0.86 & 0.95 & 0.98 & 1.00 \\
\hline & & B & 0.69 & 0.85 & 0.91 & 0.97 & 1.00 \\
\hline & Spearman & PL & 0.22 & 0.31 & 0.42 & 0.55 & 0.70 \\
\hline & & RPL & 0.26 & 0.45 & 0.67 & 0.77 & 0.87 \\
\hline & & B & 0.56 & 0.72 & 0.88 & 0.96 & 0.99 \\
\hline & Kendall & PL & 0.15 & 0.25 & 0.39 & 0.53 & 0.70 \\
\hline & & RPL & 0.26 & 0.38 & 0.56 & 0.67 & 0.81 \\
\hline & & B & 0.53 & 0.65 & 0.79 & 0.86 & 0.90 \\
\hline
\end{tabular}

Table 6: Estimated probability from 100 simulations that the optimal sequence is found in an initial random sample of $N$ sequences followed by the top $M$ sequences under various surrogates: $\mathrm{PL}=$ Plackett-Luce, $\mathrm{RPL}=$ Reverse Plackett-Luce, $\mathrm{B}=$ Benter.

better than using the other two correlation functions. Within the results for the Pearson correlation, the surrogate based on the Benter model $(\hat{f}(\cdot))$, is almost uniformly better than the other two models (PL and RPL), and provides a noticeable improvement for small $N$ and $M$. The results justify our preference for the Benter model over the PL or RPL models as the basis for a parametric surrogate. Due to its dominance in the simulations, in what follows we restrict attention to the combination of 
Benter and Pearson.

\subsection{Guidelines on choice of $N$ for a given budget $B$}

Table 6 provides some guidelines on the choices of $N$ and $M$ for problems such as this, which are useful if we have a specific budget of $B=N+M$ sequences for which we can calculate the expected utility. For example, with a budget of $B=150$ sequences, the probability that we obtain the optimal sequence is approximately $85 \%$ when $N=50, M=100$, whereas with $N=$ $100, M=50$ this probability is approximately $94 \%$.

The results in Table 6 relate to one specific "problem scenario" as defined through the values of $J, I, R_{0}, \epsilon, \lambda, p, q$ and so on, and are perhaps too narrowly focused to provide generic advice. A more extensive simulation study was carried out in order to offer more generic guidance on the choice of $N$ for a given $B$, over a broad range of "problem scenarios". A total of 10000 randomly generated "problem scenarios" were analysed in the second simulation study and the results are described in detail in the Supplementary Material. Specifically, we fit a logistic regression model with response variable an indicator of whether the optimal sequence was found for that problem scenario and with explanatory variables equal to $N, B$ and functions thereof. This approach allows us to predict the probability of finding the optimal sequence for combinations of $N$ and $B$, to approximate the optimal choice of $N$ for a given budget $B$ and predict the probability of obtaining the optimal sequence for that choice. Based on the results of this second simulation study we would recommend the rule of thumb of setting $N=M$, or, in other words, setting $N=B / 2$ (to the nearest integer). This provides a simple, default first choice in the absence of other information. Our simulations suggest that this is only marginally suboptimal, as well as being very simple and easy to remember.

Additionally, we have used the simulation results from Table 6 to determine an optimal choice of $N$ for a given budget $B$ for the specific "problem scenario" considered therein. Specifically, following the logistic regression procedure outlined in the Supplementary Material, the results suggest that, if $B=100$, which was used in the illustrative example of Section 4, the optimal choice of $N$ is 58 (and therefore $M=42$ ); this gives a probability of obtaining the optimal sequence of about $75 \%$. This is very similar to our generic rule of thumb which would suggest a 50-50 split between 
$N$ and $M$ and a probability of obtaining the optimal sequence of just over $70 \%$ (as determined by the results in the Supplememtary Material).

\subsection{Median rank performance}

Whilst we may not obtain the optimal sequence in our $N+M$ sequences it is interesting to know how the best ranked sequence in our sample compares to the full set of sequences. In each of our simulations in the first simulation study (detailed in Section 5.1) we computed the rank in terms of expected utilities of the highest ranked sequence out of our sample of $N+M$ sequences for the values of $N$ and $M$. The medians over 100 simulations of these ranks are given in Table 7 . We

\begin{tabular}{rccccc}
\hline \hline & \multicolumn{5}{c}{$M$} \\
\cline { 2 - 6 }$N$ & 10 & 20 & 50 & 100 & 200 \\
\hline 25 & 93 & 37 & 13 & 1 & 1 \\
50 & 37 & 1 & 1 & 1 & 1 \\
75 & 3 & 1 & 1 & 1 & 1 \\
100 & 1 & 1 & 1 & 1 & 1 \\
200 & 1 & 1 & 1 & 1 & 1 \\
\hline \hline
\end{tabular}

Table 7: Median from 100 simulations of the rank in terms of expected utilities of the highest ranked sequence out of our sample of $B=N+M$ sequences for the emulator based on the Benter model with maximised Pearson correlation.

see that even in very small sample sizes we do well at choosing a good sequence, and for most combinations of $N$ and $M$ we get the optimal sequence at least $50 \%$ of the time.

\subsection{Dealing with large numbers of permutations}

In our examples and simulation studies we have considered a fairly small number of tasks (up to $J=10$ ) so that we could evaluate the expected utility for all $J$ ! sequences and solve the decision problem exactly. This also means that we were able to evaluate the surrogate function at all possible sequences and find the top $M$ candidates for evaluating the expected utility. If we were interested in sequencing a larger number of tasks, say $J=20$, it may be infeasible to evaluate the surrogate function at all $J$ ! sequences. One advantage of an emulator based on a probabilistic model is that we can sample a large number of sequences (though considerably less that $J$ !) from the fitted Benter model, evaluate the surrogate function at these values, and then pick the top $M$ as the candidates to evaluate the expected utility. This simulation-based approach should scale up well to very large $J$, although such values are unlikely in reliability growth. 


\section{Summary and further work}

We have considered the emulation of utility functions for permutations. We proposed an approach based on the Benter model and have shown this can provide a good approximation in reliability growth decision making. A simple extension would be to consider the case where some tasks can be carried out in parallel.

Our proposed emulator is a unimodal function of its inputs. In the illustrative example of Section 4 the utility function was unimodal, and for the types of reliability growth tasks we consider here we expect the utility function to be largely unimodal, and so our proposed emulator is ideally suited. More generally, for mutli-modal utility functions, our proposed emulator will emulate around only one of the modes. As such, the development of a multi-modal surrogate based on a mixture of Benter models [Gormley and Murphy, 2008, Mollica and Tardella, 2014] may be worth investigating.

We chose the training set of $N$ sequences randomly. Future work would be to choose this training sample informatively, to focus the training sample in the areas of high expected utility. The ratio of benefit to cost of tasks would be useful here. The approach taken for reliability growth, maximising the expected utility of a multi-attribute utility function, can have wider use in problems in which actions are to be ordered. A future direction is to use the approach in optimal sequencing of actions in project risk management.

We emphasise that the results presented in Sections 4 and 5 are based on a simple random sample from the set of all sequences and that other more intelligent ways to explore the design space may lead to different results in terms of optimal choices of $N$ and $M$. Nevertheless this procedure allows us to assess the performance of the different surrogates and we suspect that the main messages from our simulation study — that the Benter model is preferred to the PL or RPL models, and the Pearson correlation is the superior form of objective function - will persist with other types of training sample. This has been our experience in some preliminary investigations. Our proposed procedure has similarities to an efficient single-step cross-entropy optimization algorithm [Rubinstein and Kroese, 2004]. Simulation results (given in the Supplementary Material) using a training sample derived from two steps of the cross-entropy optimization algorithm (that is, with training sequences sampled from the fitted model from the first stage) provide similar conclusions to those 
presented here. The results in terms of the probability of discovering the optimal sequence in the $N+M$ sequences are no better than those presented here for large values of $N$ and $M$, and are typically poorer for small values of $N$ and $M$. A more detailed investigation of design issues is the subject of future work.

\section{References}

W. Benter. Computer-based horse race handicapping and wagering systems: A report. In D.B. Hausch, V.S.Y. Lo, and W.T. Ziemba, editors, Efficiency of Racetrack Betting Markets, pages 183-198. Academic Press, San Diego, 1994.

W.R. Blischke and D.N.P Murthy. Reliability: Modeling, Prediction, and Optimization. Wiley Series in Probability and Statistics. Wiley, 2011. ISBN 9781118150474.

M. Caserta and S. Voß. An exact algorithm for the reliability redundancy allocation problem. European Journal of Operational Research, 244(1):110-116, 2015.

L.H. Crow. Reliability analysis of complex repairable systems. SIAM, 1974.

A.P. Eigbe, B.J. Sauser, and W. Felder. Systemic analysis of the critical dimensions of project management that impact test and evaluation program outcomes. International Journal of Project Management, 33(4):747-759, 2015.

M.A. Fligner and J.S. Verducci. Multistage ranking models. Journal of the American Statistical Association, 83:892-901, 1988.

I.C. Gormley and T.B. Murphy. Exploring voting blocs within the Irish electorate: a mixture modeling approach. Journal of the American Statistical Association, 103:1014-1027, 2008.

S. Guikema and M.E. Pate-Cornell. Component choice for managing risk in engineered systems with generalized risk/cost functions. Reliability Engineering and System Safety, 78:227-238, 2002.

D.A. Henderson, R.J. Boys, K.J. Krishnan, C. Lawless, and D.J. Wilkinson. Bayesian emulation and calibration of a stochastic computer model of mitochondrial dna deletions in substantia nigra neurons. Journal of the American Statistical Association, 104:76-87, 2009.

C. Hsieh. Optimal task allocation and hardware redundancy policies in distributed computing systems. European Journal of Operational Research, 147:430-447, 2003.

C. Hsieh and Y. Hsieh. Reliability and cost optimization in distribution computing systems. Computers and Operations Research, 30:1103-1119, 2003.

W. Johnston, J. Quigley, and L. Walls. Optimal allocation of reliability tasks to mitigate faults during system development. IMA Journal of Management Mathematics, 17:159-169, 2006.

D.R. Jones, M. Schonlau, and W.J. Welch. Efficient global optimization of expensive black-box functions. Journal of Global Optimization, 13:455-492, 1998.

M. Kennedy and A. O'Hagan. Bayesian calibration of computer models (with discussion). J. R. Statist. Soc. Ser. B, 63: 425-464, 2001.

Y.H. Kim, A. Moraglio, A. Kattan, and Y. Yoon. Geometric generalisation of surrogate model-based optimisation to combinatorial and program spaces. Mathematical Problems in Engineering, 2014, 2014.

K. Knight. Mathematical Statistics. Chapman and Hall, 2000.

M. Krasich. Modeling of sw reliability in early design with planning and measurement of its reliability growth. In Reliability and Maintainability Symposium (RAMS), 2015 Annual, pages 1-6. IEEE, 2015.

M. Krasich, J. Quigley, and L. Walls. Modeling reliability growth in the product design process. In Reliability And Maintainability, 2004 Annual Symposium-RAMS, pages 424-430. IEEE, 2004.

G. Levitin, L. Xing, S. Peng, and Y. Dai. Optimal choice of standby modes in 1-out-of-n system with respect to mission reliability and cost. Applied Mathematics and Computation, 258:587-596, 2015.

R.D. Luce. Individual Choice Behavior. Wiley, New York, 1959.

J.I. Marden. Analysing and Modeling rank data. Chapman and Hall, London, 1995.

C. Mollica and L. Tardella. Epitope profiling via mixture modelling of ranked data. Statistics in Medicine, 33:3738-3758, 2014.

A. Moraglio and A. Kattan. Geometric generalisation of surrogate model-based optimisation to combinatorial spaces. In Evolutionary Computation in Combinatorial Optimization, pages 142-154. Springer, 2011.

A. Moraglio, Y.H. Kim, and Y. Yoon. Geometric surrogate-based optimisation for permutation-based problems. In Proceedings of the 13th Annual Conference Companion on Geometric and Evolutionary Computation, pages 133134. ACM, 2011.

P. Müller and G. Parmigiani. Optimal design via curbe fitting of Monte Carlo experiments. Journal of the American Statistical Association, 90:1322-1330, 1995. 
J.A. Nelder and R. Mead. A simplex method for function minimization. Computer Journal, 7:308-313, 1965.

P.D.T. O’Connor and A. Kleyner. Practical reliability engineering. John Wiley \& Sons, 2012.

R. L. Plackett. The analysis of permutations. Applied Statistics, 24:193-202, 1975.

J. Quigley and L. Walls. Measuring the effectiveness of reliability growth testing. Quality and Reliability Engineering, 15:87-93, 1999.

J. Quigley and L. Walls. Confidence intervals for reliability growth models with small sample sizes. IEEE Transactions on Reliability, 52:257-262, 2003.

J. Quigley and L. Walls. Trading reliability targets within a supply chain using Shapley's value. Reliability Engineering and System Safety, 92:1448-1457, 2006.

R Core Team. R: A Language and Environment for Statistical Computing. R Foundation for Statistical Computing, Vienna, Austria, 2014.

R.Y. Rubinstein and D.P. Kroese. The Cross-Entropy Method: A Unified Approach to Combinatorial Optimization, Monte-Carlo Simulation, and Machine Learning. Springer-Verlag, New York, 2004.

J. Sacks, W.J. Welch, T.J. Mitchell, and H.P. Wynn. Design and analysis of computer experiments. Statistical Science, 4:409-423, 1989.

M. Silverman. Summary of halt and hass results at an accelerated reliability test center. In Reliability and Maintainability Symposium, 1998. Proceedings., Annual. IEEE, 1998.

U.S. Department of Defense. Defense science board task force report on developmental test and evaluation. Technical report, 2008.

U.S. Department of Defense. Directive-type memorandum (dtm) 11-003 - reliability analysis, planning, tracking, and reporting. Technical report, 2011.

I. Voutchkov, A.J. Keane, A. Bhaskar, and T.M. Olsen. Weld sequence optimization: The use of surrogate models for solving combinatorial problems. Computational Methods in Applied Mechanics and Engineering, 194:3535-3551, 2005.

L. Walls and J. Quigley. Learning to improve reliability during system development. European Journal of Operational Research, 119:495-509, 1999.

L. Walls and J. Quigley. Building prior distributions to support bayesian reliability growth modelling using expert judgement. Reliability Engineering and System Safety, 74:117-128, 2001.

L. Walls, J. Quigley, and M. Kraisch. Comparison of two models for managing reliability growth during product development. IMA Journal of Mathematics Applied in Business and Industry, 16:12-22, 2005.

M. Wayne and M. Modarres. A bayesian model for complex system reliability growth under arbitrary corrective actions. Reliability, IEEE Transactions on, 64(1):206-220, 2015.

K.J. Wilson and J. Quigley. Allocation of tasks for reliability growth using multi-attribute utility. European Journal of Operational Research, 255:259-271, 2016.

P.C. Young and M. Ratto. Statistical emulation of large linear dynamic models. Technometrics, 53(1):29-43, 2011.

M. Zaefferer. Package 'CEGO'. https : / cran.r-project.org/web/packages/CEGO/CEGO.pdf, 2015.

M. Zaefferer, J. Stork, and T. Bartz-Beielstein. Distance measures for permutations in combinatorial efficient global optimization. In Parallel Problem Solving from Nature - PPSN XIII, volume 8672 of Lecture Notes in Computer Science, pages 373-383. Springer, 2014a.

M. Zaefferer, J. Stork, M. Friese, A. Fischbach, B. Naujoks, and T. Bartz-Beielstein. Efficient global optimization for combinatorial problems. In Proceedings of the 2014 Annual Conference on Genetic and Evolutionary Computation, pages 871-878. ACM, 2014b.

Q. Zhou, P.Z.G. Qian, and S. Zhou. A simple approach to emulation for computer models with qualitative and quantitative factors. Technometrics, 53(3):266-273, 2011. 